\title{
Nonlocal Hadamard fractional integral conditions for nonlinear Riemann-Liouville fractional differential equations
}

\author{
Jessada Tariboon ${ }^{1 *}$, Sotiris K Ntouyas ${ }^{2}$ and Weerawat Sudsutad ${ }^{1}$
}

\section{"Correspondence:}

jessadat@kmutnb.ac.th

${ }^{1}$ Nonlinear Dynamic Analysis

Research Center, Department of

Mathematics, Faculty of Applied

Science, King Mongkut's University

of Technology North Bangkok,

Bangkok, 10800, Thailand

Full list of author information is

available at the end of the article

\begin{abstract}
In this paper, we introduce a new class of boundary value problems consisting of a fractional differential equation of Riemann-Liouville type, ${ }_{R L} D^{q} x(t)=f(t, x(t)), t \in[0, T]$, subject to the Hadamard fractional integral conditions $x(0)=0, x(T)=\sum_{i=1}^{n} \alpha_{i H^{1}} P_{i} x\left(\eta_{i}\right)$. Existence and uniqueness results are obtained by using a variety of fixed point theorems. Examples illustrating the results obtained are also presented.
\end{abstract}

MSC: 34A08; 34B15

Keywords: Riemann-Liouville fractional derivative; Hadamard fractional integral; existence; uniqueness; fixed point theorems

\section{Introduction}

In this paper, we concentrate on the study of existence and uniqueness of solutions for the nonlinear Riemann-Liouville fractional differential equation with nonlocal Hadamard fractional integral boundary conditions of the form

$$
\begin{aligned}
& { }_{R L} D^{q} x(t)=f(t, x(t)), \quad t \in[0, T], \\
& x(0)=0, \quad x(T)=\sum_{i=1}^{n} \alpha_{i H} I^{p_{i}} x\left(\eta_{i}\right),
\end{aligned}
$$

where $1<q \leq 2,{ }_{R L} D^{q}$ is the standard Riemann-Liouville fractional derivative of order $q$, ${ }_{H} I^{p_{i}}$ is the Hadamard fractional integral of order $p_{i}>0, \eta_{i} \in(0, T), f:[0, T] \times \mathbb{R} \rightarrow \mathbb{R}$, and $\alpha_{i} \in \mathbb{R}, i=1,2, \ldots, n$ are real constants such that $\sum_{i=1}^{n} \frac{\alpha_{i} \eta_{i}^{q-1}}{(q-1)^{p_{i}}} \neq T^{q-1}$.

Several interesting and important results concerning existence and uniqueness of solutions, stability properties of solutions, analytic and numerical methods of solutions for fractional differential equations can be found in the recent literature on the topic and the search for more and more results is in progress. Fractional-order operators are nonlocal in nature and take care of the hereditary properties of many phenomena and processes. Fractional calculus has also emerged as a powerful modeling tool for many real world problems. For examples and recent development of the topic, see [1-14]. However, it has been observed that most of the work on the topic involves either Riemann-Liouville or Caputo type fractional derivatives. Besides these derivatives, the Hadamard fractional derivative is another kind of fractional derivative that was introduced by Hadamard in 1892 [15]. 
This fractional derivative differs from the other ones in the sense that the kernel of the integral (in the definition of the Hadamard derivative) contains a logarithmic function of an arbitrary exponent. For background material of the Hadamard fractional derivative and integral, we refer to $[2,16-22]$.

In the present paper we initiate the study of boundary value problems like (1.1)-(1.2), in which we combine Riemann-Liouville fractional differential equations subject to the Hadamard fractional integral boundary conditions. The key tool for this combination is Property 2.25 from [2], p.113. To the best of the authors' knowledge this is the first paper dealing with the Riemann-Liouville fractional differential equation subject to Hadamard type integral boundary conditions.

Several new existence and uniqueness results are obtained by using a variety of fixed point theorems. Thus, in Theorem 3.1 we present an existence and uniqueness result via Banach's fixed point theorem, while in Theorems 3.2 and 3.3 we give two other existence and uniqueness results via Banach's fixed point theorem and Hölder inequality and nonlinear contractions, respectively. In the sequel existence results are obtained in Theorem 3.4, via Krasnoselskii's fixed point theorem, in Theorem 3.5 via Leray-Schauder's nonlinear alternative and finally in Theorem 3.7 via Leray-Schauder's degree theory. Examples illustrating the results obtained are also presented.

\section{Preliminaries}

In this section, we introduce some notations and definitions of fractional calculus and present preliminary results needed in our proofs later.

Definition 2.1 The Riemann-Liouville fractional derivative of order $q>0$ of a continuous function $f:(0, \infty) \rightarrow \mathbb{R}$ is defined by

$$
{ }_{R L} D^{q} f(t)=\frac{1}{\Gamma(n-q)}\left(\frac{d}{d t}\right)^{n} \int_{0}^{t}(t-s)^{n-q-1} f(s) d s, \quad n-1<q<n,
$$

where $n=[q]+1,[q]$ denotes the integer part of a real number $q$. Here $\Gamma$ is the Gamma function defined by $\Gamma(q)=\int_{0}^{\infty} e^{-s} s^{q-1} d s$.

Definition 2.2 The Riemann-Liouville fractional integral of order $q>0$ of a continuous function $f:(0, \infty) \rightarrow \mathbb{R}$ is defined by

$$
{ }_{R L} I^{q} f(t)=\frac{1}{\Gamma(q)} \int_{0}^{t}(t-s)^{q-1} f(s) d s
$$

Definition 2.3 The Hadamard derivative of fractional order $q$ for a function $f:(0, \infty) \rightarrow$ $\mathbb{R}$ is defined as

$$
{ }_{H} D^{q} f(t)=\frac{1}{\Gamma(n-q)}\left(t \frac{d}{d t}\right)^{n} \int_{0}^{t}\left(\log \frac{t}{s}\right)^{n-q-1} \frac{f(s)}{s} d s, \quad n-1<q<n, n=[q]+1,
$$

where $\log (\cdot)=\log _{e}(\cdot)$, provided the integral exists. 
Definition 2.4 The Hadamard fractional integral of order $q \in \mathbb{R}^{+}$of a function $f(t)$, for all $t>0$, is defined as

$$
{ }_{H} I^{q} f(t)=\frac{1}{\Gamma(q)} \int_{0}^{t}\left(\log \frac{t}{s}\right)^{q-1} f(s) \frac{d s}{s}
$$

provided the integral exists.

Lemma $2.1([2, \mathrm{p} .113])$ Let $q>0$ and $n>0$. Then the following formulas:

$$
\left({ }_{H} I^{q} s^{n}\right)(t)=n^{-q} t^{n} \quad \text { and } \quad\left({ }_{H} D^{q} s^{n}\right)(t)=n^{q} t^{n}
$$

hold.

Lemma 2.2 Let $q>0$ and $x \in C(0, T) \cap L(0, T)$. Then the fractional differential equation

$$
{ }_{R L} D^{q} x(t)=0
$$

has a unique solution

$$
x(t)=c_{1} t^{q-1}+c_{2} t^{q-2}+\cdots+c_{n} t^{q-n},
$$

where $c_{i} \in \mathbb{R}, i=1,2, \ldots, n$, and $n-1<q<n$.

Lemma 2.3 Let $q>0$. Then for $x \in C(0, T) \cap L(0, T)$ we have

$$
{ }_{R L} I^{q}{ }_{R L} D^{q} x(t)=x(t)+c_{1} t^{q-1}+c_{2} t^{q-2}+\cdots+c_{n} t^{q-n},
$$

where $c_{i} \in \mathbb{R}, i=1,2, \ldots, n$, and $n-1<q<n$.

Lemma 2.4 Let $\sum_{i=1}^{n}\left(\left(\alpha_{i} \eta_{i}^{q-1}\right) /\left((q-1)^{p_{i}}\right)\right) \neq T^{q-1}, 1<q \leq 2, p_{i}>0, \alpha_{i} \in \mathbb{R}, \eta_{i} \in(0, T), i=$ $1,2,3, \ldots, n$, and $h \in C([0, T], \mathbb{R})$. Then the nonlocal Hadamard fractional integral problem for the nonlinear Riemann-Liouville fractional differential equation

$$
{ }_{R L} D^{q} x(t)=h(t), \quad 0<t<T,
$$

subject to the boundary conditions

$$
x(0)=0, \quad x(T)=\sum_{i=1}^{n} \alpha_{i H} I^{p_{i}} x\left(\eta_{i}\right),
$$

has a unique solution given by

$$
x(t)={ }_{R L} I^{q} h(t)-\frac{t^{q-1}}{\lambda}\left({ }_{R L} I^{q} h(T)-\sum_{i=1}^{n} \alpha_{i}\left({ }_{H} I^{p_{i}}{ }_{R L} I^{q} h\right)\left(\eta_{i}\right)\right),
$$

where

$$
\lambda:=T^{q-1}-\sum_{i=1}^{n} \frac{\alpha_{i} \eta_{i}^{q-1}}{(q-1)^{p_{i}}} \neq 0 .
$$


Proof Using Lemmas 2.2-2.3, (2.1) can be expressed as an equivalent integral equation

$$
x(t)={ }_{R L} I^{q} h(t)-c_{1} t^{q-1}-c_{2} t^{q-2},
$$

for $c_{1}, c_{2} \in \mathbb{R}$. The first condition of (2.2) implies that $c_{2}=0$. Taking the Hadamard fractional integral of order $p_{i}>0$ for (2.5) and using the property of the Hadamard fractional integral $\left({ }_{H} I^{p_{i}} s^{q-1}\right)(t)=(q-1)^{-p_{i}} t^{q-1}$, we get

$$
{ }_{H} I^{p_{i}} x(t)=\left({ }_{H} I^{p_{i}}{ }_{R L} I^{q} h\right)(t)-c_{1}\left(H^{P_{i}} s^{q-1}\right)(t)=\left({ }_{H} I^{p_{i}}{ }_{R L} I^{q} h\right)(t)-c_{1} \frac{t^{q-1}}{(q-1)^{p_{i}}} .
$$

The second condition of (2.2) implies that

$$
{ }_{R L} I^{q} h(T)-c_{1} T^{q-1}=\sum_{i=1}^{n} \alpha_{i}\left({ }_{H} I^{p_{i}}{ }_{R L} I^{q} h\right)\left(\eta_{i}\right)-c_{1} \sum_{i=1}^{n} \frac{\alpha_{i} \eta_{i}^{q-1}}{(q-1)^{p_{i}}} .
$$

Thus,

$$
c_{1}=\frac{1}{\lambda}\left({ }_{R L} I^{q} h(T)-\sum_{i=1}^{n} \alpha_{i}\left({ }_{H} I^{p_{i}}{ }_{R L} I^{q} h\right)\left(\eta_{i}\right)\right) .
$$

Substituting the values of $c_{1}$ and $c_{2}$ in (2.5), we obtain the solution (2.3).

\section{Main results}

Throughout this paper, for convenience, we use the following expressions:

$$
{ }_{R L} I^{\alpha} f(s, x(s))(z)=\frac{1}{\Gamma(\alpha)} \int_{0}^{z}(z-s)^{\alpha-1} f(s, x(s)) d s, \quad z \in\{t, T\},
$$

for $t \in[0, T]$ and

$$
{ }_{H} I^{p_{i}}{ }_{R L} I^{\alpha} f(s, x(s))\left(\eta_{i}\right)=\frac{1}{\Gamma\left(p_{i}\right) \Gamma(\alpha)} \int_{0}^{\eta_{i}} \int_{0}^{r}\left(\log \frac{\eta_{i}}{r}\right)^{p_{i}-1}(r-s)^{\alpha-1} \frac{f(s, x(s))}{r} d s d r,
$$

where $\eta_{i} \in(0, T)$ for $i=1,2, \ldots, n$.

Let $\mathcal{C}=C([0, T], \mathbb{R})$ denote the Banach space of all continuous functions from $[0, T]$ to $\mathbb{R}$ endowed with the norm defined by $\|x\|=\sup _{t \in[0, T]}|x(t)|$. As in Lemma 2.4, we define an operator $\mathcal{A}: \mathcal{C} \rightarrow \mathcal{C}$ by

$$
\begin{aligned}
(\mathcal{A} x)(t)= & { }_{R L} I^{q} f(s, x(s))(t) \\
& -\frac{t^{q-1}}{\lambda}\left({ }_{R L} I^{q} f(s, x(s))(T)-\sum_{i=1}^{n} \alpha_{i}\left({ }_{H} I^{p_{i}}{ }_{R L} I^{q} f(s, x(s))\right)\left(\eta_{i}\right)\right) .
\end{aligned}
$$

It should be noticed that problem (1.1)-(1.2) has a solution if and only if the operator $\mathcal{A}$ has fixed points.

In the following, for the sake of convenience, we set a constant

$$
\Omega:=\frac{T^{q}}{\Gamma(q+1)}+\frac{T^{2 q-1}}{|\lambda| \Gamma(q+1)}+\frac{T^{q-1}}{|\lambda| \Gamma(q+1)} \sum_{i=1}^{n}\left|\alpha_{i}\right| q^{-p_{i}} \eta_{i}^{q} .
$$


In the following subsections we prove existence, as well as existence and uniqueness results, for the boundary value problem (1.1)-(1.2) by using a variety of fixed point theorems.

\subsection{Existence and uniqueness result via Banach's fixed point theorem}

Theorem 3.1 Assume that:

$\left(\mathrm{H}_{1}\right)$ there exists a constant $L>0$ such that $|f(t, x)-f(t, y)| \leq L|x-y|$, for each $t \in[0, T]$ and $x, y \in \mathbb{R}$.

If

$L \Omega<1$

where $\Omega$ is defined by (3.2), then the boundary value problem (1.1)-(1.2) has a unique solution on $[0, T]$.

Proof We transform the BVP (1.1)-(1.2) into a fixed point problem, $x=\mathcal{A} x$, where the operator $\mathcal{A}$ is defined as in (3.1). Observe that the fixed points of the operator $\mathcal{A}$ are solutions of problem (1.1)-(1.2). Applying the Banach contraction mapping principle, we shall show that $\mathcal{A}$ has a unique fixed point.

We let $\sup _{t \in[0, T]}|f(t, 0)|=M<\infty$, and choose

$$
r \geq \frac{M \Omega}{1-L \Omega}
$$

Now, we show that $\mathcal{A} B_{r} \subset B_{r}$, where $B_{r}=\{x \in \mathcal{C}:\|x\| \leq r\}$. For any $x \in B_{r}$, we have

$$
\begin{aligned}
|(\mathcal{A} x)(t)| \leq & \sup _{t \in[0, T]}\left\{R L I^{q}|f(s, x(s))|(t)+\frac{t^{q-1}}{|\lambda|} R L I^{q}|f(s, x(s))|(T)\right. \\
& \left.+\frac{t^{q-1}}{|\lambda|} \sum_{i=1}^{n}\left|\alpha_{i}\right|_{H} I^{p_{i}}{ }_{R L} I^{q}|f(s, x(s))|\left(\eta_{i}\right)\right\} \\
\leq & R L I^{q}(|f(s, x(s))-f(s, 0)|+|f(s, 0)|)(T) \\
& +\frac{T^{q-1}}{|\lambda|} R I^{q}(|f(s, x(s))-f(s, 0)|+|f(s, 0)|)(T) \\
& +\frac{T^{q-1}}{|\lambda|} \sum_{i=1}^{n}\left|\alpha_{i}\right|_{H} I^{p_{i}}{ }_{R L} I^{q}(|f(s, x(s))-f(s, 0)|+|f(s, 0)|)\left(\eta_{i}\right) \\
\leq & (L\|x\|+M)_{R L} I^{q}(1)(T)+(L\|x\|+M) \frac{T^{q-1}}{|\lambda|} R L I^{q}(1)(T) \\
& +(L\|x\|+M) \frac{T^{q-1}}{|\lambda|} \sum_{i=1}^{n}\left|\alpha_{i}\right|_{H} I^{p_{i}} R L I^{q}(1)\left(\eta_{i}\right) \\
= & (L r+M) \Omega \leq r, \\
= & L r+M)\left(\frac{T^{q}}{\Gamma(q+1)}+\frac{T^{2 q-1}}{|\lambda| \Gamma(q+1)}+\frac{T^{q-1}}{|\lambda| \Gamma(q+1)} \sum_{i=1}^{n}\left|\alpha_{i}\right| q^{-p_{i}} \eta_{i}^{q}\right) \\
& (L)
\end{aligned}
$$

which implies that $\mathcal{A} B_{r} \subset B_{r}$. 
Next, we let $x, y \in \mathcal{C}$. Then for $t \in[0, T]$, we have

$$
\begin{aligned}
& |(\mathcal{A} x)(t)-(\mathcal{A} y)(t)| \\
& \leq{ }_{R L} I^{q}|f(s, x(s))-f(s, y(s))|(t)+\frac{T^{q-1}}{|\lambda|} R L I^{q}|f(s, x(s))-f(s, y(s))|(T) \\
& \quad+\frac{T^{q-1}}{|\lambda|} \sum_{i=1}^{n}\left|\alpha_{i}\right|_{H} I^{p_{i}}{ }_{R L} I^{q}|f(s, x(s))-f(s, y(s))|\left(\eta_{i}\right) \\
& \leq L\left(\frac{T^{q}}{\Gamma(q+1)}+\frac{T^{2 q-1}}{|\lambda| \Gamma(q+1)}+\frac{T^{q-1}}{|\lambda| \Gamma(q+1)} \sum_{i=1}^{n}\left|\alpha_{i}\right| q^{-p_{i}} \eta_{i}^{q}\right)\|x-y\| \\
& =L \Omega\|x-y\|
\end{aligned}
$$

which implies that $\|\mathcal{A} x-\mathcal{A} y\| \leq L \Omega\|x-y\|$. As $L \Omega<1, \mathcal{A}$ is a contraction. Therefore, we deduce, by the Banach contraction mapping principle, that $\mathcal{A}$ has a fixed point which is the unique solution of the boundary value problem (1.1)-(1.2). The proof is completed.

\subsection{Existence and uniqueness result via Banach's fixed point theorem and Hölder inequality}

Theorem 3.2 Suppose that: $f:[0, T] \times \mathbb{R} \rightarrow \mathbb{R}$ is a continuous function satisfying the following assumption:

$\left(\mathrm{H}_{2}\right)|f(t, x)-f(t, y)| \leq \delta(t)|x-y|$, for $t \in[0, T], x, y \in \mathbb{R}$ and $\delta \in L^{\frac{1}{\sigma}}\left([0, T], \mathbb{R}^{+}\right), \sigma \in(0,1)$.

Denote $\|\delta\|=\left(\int_{0}^{T}|\delta(s)|^{\frac{1}{\sigma}} d s\right)^{\sigma}$. If

$$
\begin{gathered}
\|\delta\|\left\{\frac{T^{q-\sigma}}{\Gamma(q)}\left(\frac{1-\sigma}{q-\sigma}\right)^{1-\sigma}+\frac{T^{2 q-\sigma-1}}{|\lambda| \Gamma(q)}\left(\frac{1-\sigma}{q-\sigma}\right)^{1-\sigma}\right. \\
\left.+\frac{T^{q-1}}{|\lambda| \Gamma(q)}\left(\frac{1-\sigma}{q-\sigma}\right)^{1-\sigma} \sum_{i=1}^{n}\left|\alpha_{i}\right|(q-\sigma)^{p_{i}} \eta_{i}^{q-\sigma}\right\}<1,
\end{gathered}
$$

then the boundary value problem (1.1)-(1.2) has a unique solution.

Proof For $x, y \in C([0, T], \mathbb{R})$ and for each $t \in[0, T]$, by Hölder's inequality, we have

$$
\begin{aligned}
|(\mathcal{A} x)(t)-(\mathcal{A} y)(t)| & \\
\leq & R L I^{q}|f(s, x(s))-f(s, y(s))|(t)+\frac{T^{q-1}}{|\lambda|} R L I^{q}|f(s, x(s))-f(s, y(s))|(T) \\
& +\frac{T^{q-1}}{|\lambda|} \sum_{i=1}^{n}\left|\alpha_{i}\right|_{H} I^{p_{i}}{ }_{R L} I^{q}|f(s, x(s))-f(s, y(s))|\left(\eta_{i}\right) \\
= & \frac{1}{\Gamma(q)} \int_{0}^{t}(t-s)^{q-1} \delta(s)|x(s)-y(s)| d s+\frac{T^{q-1}}{|\lambda| \Gamma(q)} \int_{0}^{T}(T-s)^{q-1} \delta(s)|x(s)-y(s)| d s \\
& +\frac{T^{q-1}}{|\lambda|} \sum_{i=1}^{n} \frac{\left|\alpha_{i}\right|}{\Gamma\left(p_{i}\right) \Gamma(q)} \int_{0_{+}}^{\eta_{i}} \int_{0_{+}}^{s}\left(\log \frac{\eta_{i}}{s}\right)^{p_{i}-1}(s-r)^{q-1} \delta(r)|x(r)-y(r)| d r \frac{d s}{s} \\
\leq & \frac{1}{\Gamma(q)}\left(\int_{0}^{t}\left((t-s)^{q-1}\right)^{\frac{1}{1-\sigma}} d s\right)^{1-\sigma}\left(\int_{0}^{t}(\delta(s))^{\frac{1}{\sigma}} d s\right)^{\sigma}\|x-y\|
\end{aligned}
$$




$$
\begin{aligned}
& +\frac{T^{q-1}}{|\lambda| \Gamma(q)}\left(\int_{0}^{T}\left((T-s)^{q-1}\right)^{\frac{1}{1-\sigma}} d s\right)^{1-\sigma}\left(\int_{0}^{T}(\delta(s))^{\frac{1}{\sigma}} d s\right)^{\sigma}\|x-y\| \\
& +\frac{T^{q-1}}{|\lambda|} \sum_{i=1}^{n} \frac{\left|\alpha_{i}\right|}{\Gamma(q) \Gamma\left(p_{i}\right)} \int_{0}^{\eta_{i}}\left(\log \frac{\eta_{i}}{s}\right)^{p_{i}-1}\left(\int_{0}^{s}\left((s-r)^{q-1}\right)^{\frac{1}{1-\sigma}} d r\right)^{1-\sigma} \\
& \times\left(\int_{0}^{s}(\delta(r))^{\frac{1}{\sigma}} d r\right)^{\sigma} \frac{d s}{s}\|x-y\| \\
& \leq\|\delta\| \frac{T^{q-\sigma}}{\Gamma(q)}\left(\frac{1-\sigma}{q-\sigma}\right)^{1-\sigma}\|x-y\|+\|\delta\| \frac{T^{2 q-\sigma-1}}{|\lambda| \Gamma(q)}\left(\frac{1-\sigma}{q-\sigma}\right)^{1-\sigma}\|x-y\| \\
& +\|\delta\| \frac{T^{q-1}}{|\lambda| \Gamma(q)}\left(\frac{1-\sigma}{q-\sigma}\right)^{1-\sigma} \sum_{i=1}^{n} \frac{\left|\alpha_{i}\right|}{\Gamma\left(p_{i}\right)} \int_{0}^{\eta_{i}}\left(\log \frac{\eta_{i}}{s}\right)^{p_{i}-1} s^{q-\sigma} \frac{d s}{s}\|x-y\| \\
& \leq\|\delta\|\left[\frac{T^{q-\sigma}}{\Gamma(q)}\left(\frac{1-\sigma}{q-\sigma}\right)^{1-\sigma}+\frac{T^{2 q-\sigma-1}}{|\lambda| \Gamma(q)}\left(\frac{1-\sigma}{q-\sigma}\right)^{1-\sigma}+\frac{T^{q-1}}{|\lambda| \Gamma(q)}\left(\frac{1-\sigma}{q-\sigma}\right)^{1-\sigma}\right. \\
& \left.\quad \times \sum_{i=1}^{n}\left|\alpha_{i}\right|(q-\sigma)^{p_{i}} \eta_{i}^{q-\sigma}\right]\|x-y\| .
\end{aligned}
$$

It follows that $\mathcal{A}$ is contraction mapping. Hence Banach's fixed point theorem implies that $\mathcal{A}$ has a unique fixed point, which is the unique solution of the boundary value problem (1.1)-(1.2). The proof is completed.

\subsection{Existence and uniqueness result via nonlinear contractions}

Definition 3.1 Let $E$ be a Banach space and let $\mathcal{A}: E \rightarrow E$ be a mapping. $\mathcal{A}$ is said to be a nonlinear contraction if there exists a continuous nondecreasing function $\Psi: \mathbb{R}^{+} \rightarrow \mathbb{R}^{+}$ such that $\Psi(0)=0$ and $\Psi(\varepsilon)<\varepsilon$ for all $\varepsilon>0$ with the property:

$$
\|\mathcal{A} x-\mathcal{A} y\| \leq \Psi(\|x-y\|), \quad \forall x, y \in E .
$$

Lemma 3.1 (Boyd and Wong) [23] Let $E$ be a Banach space and let $\mathcal{A}: E \rightarrow E$ be a nonlinear contraction. Then $\mathcal{A}$ has a unique fixed point in $E$.

Theorem 3.3 Let $f:[0, T] \times \mathbb{R} \rightarrow \mathbb{R}$ be a continuous function satisfying the assumption:

$\left(\mathrm{H}_{3}\right)|f(t, x)-f(t, y)| \leq h(t) \frac{|x-y|}{H^{*}+|x-y|}$, for $t \in[0, T], x, y \geq 0$, where $h:[0, T] \rightarrow \mathbb{R}^{+}$is continuous and $H^{*}$ the constant defined by

$$
H^{*}:={ }_{R L} I^{q} h(T)+\frac{T^{q-1}}{|\lambda|} R L I^{q} h(T)+\frac{T^{q-1}}{|\lambda|} \sum_{i=1}^{n}\left|\alpha_{i}\right|_{H} I^{p_{i}}{ }_{R L} I^{q} h\left(\eta_{i}\right) .
$$

Then the boundary value problem (1.1)-(1.2) has a unique solution.

Proof We define the operator $\mathcal{A}: \mathcal{C} \rightarrow \mathcal{C}$ as in (3.1) and the continuous nondecreasing function $\Psi: \mathbb{R}^{+} \rightarrow \mathbb{R}^{+}$by

$$
\Psi(\varepsilon)=\frac{H^{*} \varepsilon}{H^{*}+\varepsilon}, \quad \forall \varepsilon \geq 0
$$

Note that the function $\Psi$ satisfies $\Psi(0)=0$ and $\Psi(\varepsilon)<\varepsilon$ for all $\varepsilon>0$. 
For any $x, y \in \mathcal{C}$ and for each $t \in[0, T]$, we have

$$
\begin{aligned}
&|(\mathcal{A} x)(t)-(\mathcal{A} y)(t)| \\
& \leq R_{R L} I^{q}|f(s, x(s))-f(s, y(s))|(t)+\frac{T^{q-1}}{|\lambda|} R I^{q}|f(s, x(s))-f(s, y(s))|(T) \\
& \quad+\frac{T^{q-1}}{|\lambda|} \sum_{i=1}^{n}\left|\alpha_{i}\right|_{H} I^{p_{i}}{ }_{R L} I^{q}|f(s, x(s))-f(s, y(s))|\left(\eta_{i}\right) \\
& \leq R_{R L} I^{q}\left(h(s) \frac{|x-y|}{H^{*}+|x-y|}\right)(T)+\frac{T^{q-1}}{|\lambda|} R L I^{q}\left(h(s) \frac{|x-y|}{H^{*}+|x-y|}\right)(T) \\
& \quad+\frac{T^{q-1}}{|\lambda|} \sum_{i=1}^{n}\left|\alpha_{i}\right|_{H} I^{p_{i}}{ }_{R L} I^{q}\left(h(s) \frac{|x-y|}{H^{*}+|x-y|}\right)\left(\eta_{i}\right) \\
& \leq \frac{\Psi(\|x-y\|)}{H^{*}}\left({ }_{R L} I^{q} h(T)+\frac{T^{q-1}}{|\lambda|} R L I^{q} h(T)+\frac{T^{q-1}}{|\lambda|} \sum_{i=1}^{n}\left|\alpha_{i}\right|_{H} I^{p_{i}}{ }_{R L} I^{q} h\left(\eta_{i}\right)\right) \\
&= \Psi(\|x-y\|) .
\end{aligned}
$$

This implies that $\|\mathcal{A} x-\mathcal{A} y\| \leq \Psi(\|x-y\|)$. Therefore $\mathcal{A}$ is a nonlinear contraction. Hence, by Lemma 3.1 the operator $\mathcal{A}$ has a unique fixed point which is the unique solution of the boundary value problem (1.1)-(1.2). This completes the proof.

\subsection{Existence result via Krasnoselskii's fixed point theorem}

Lemma 3.2 (Krasnoselskii's fixed point theorem) [24] Let M be a closed, bounded, convex, and nonempty subset of a Banach space X. Let $A, B$ be the operators such that (a) $A x+B x \in$ $M$ whenever $x, y \in M$; (b) $A$ is compact and continuous; (c) $B$ is a contraction mapping. Then there exists $z \in M$ such that $z=A z+B z$.

Theorem 3.4 Let $f:[0, T] \times \mathbb{R} \rightarrow \mathbb{R}$ be a continuous function satisfying $\left(\mathrm{H}_{1}\right)$. In addition we assume that:

$\left(\mathrm{H}_{4}\right)|f(t, x)| \leq \varphi(t), \forall(t, x) \in[0, T] \times \mathbb{R}$, and $\varphi \in C\left([0, T], \mathbb{R}^{+}\right)$.

Then the boundary value problem (1.1)-(1.2) has at least one solution on [0,T] provided

$$
L\left(\frac{T^{2 q-1}}{|\lambda| \Gamma(q+1)}+\frac{T^{q-1}}{|\lambda| \Gamma(q+1)} \sum_{i=1}^{n}\left|\alpha_{i}\right| q^{-p_{i}} \eta_{i}^{q}\right)<1 .
$$

Proof Setting $\sup _{t \in[0, T]} \varphi(t)=\|\varphi\|$ and choosing

$$
\rho \geq\|\varphi\| \Omega
$$

(where $\Omega$ is defined by (3.2)), we consider $B_{\rho}=\{x \in \mathcal{C}([0, T], \mathbb{R}):\|x\| \leq \rho\}$. We define the operators $\mathcal{A}_{1}$ and $\mathcal{A}_{2}$ on $B_{\rho}$ by

$$
\begin{aligned}
& \mathcal{A}_{1} x(t)={ }_{R L} I^{q} f(s, x(s))(t), \quad t \in[0, T], \\
& \mathcal{A}_{2} x(t)=-\frac{t^{q-1}}{\lambda}\left({ }_{R L} I^{q} f(s, x(s))(T)-\sum_{i=1}^{n} \alpha_{i}\left({ }_{H} I^{p_{i}}{ }_{R L} I^{q} f(s, x(s))\right)\left(\eta_{i}\right)\right), \quad t \in[0, T] .
\end{aligned}
$$


For any $x, y \in B_{\rho}$, we have

$$
\begin{aligned}
& \left|\left(\mathcal{A}_{1} x\right)(t)+\left(\mathcal{A}_{2} y\right)(t)\right| \\
& \leq \sup _{t \in[0, T]}\left\{R L I^{q}|f(s, x(s))|(t)+\frac{t^{q-1}}{|\lambda|} R L^{q}|f(s, y(s))|(T)\right. \\
& \left.\quad+\frac{t^{q-1}}{|\lambda|} \sum_{i=1}^{n}\left|\alpha_{i}\right|_{H} I^{p_{i}} I_{L} I^{q}|f(s, y(s))|\left(\eta_{i}\right)\right\} \\
& \leq\|\varphi\|\left(\frac{T^{q}}{\Gamma(q+1)}+\frac{T^{2 q-1}}{|\lambda| \Gamma(q+1)}+\frac{T^{q-1}}{|\lambda| \Gamma(q+1)} \sum_{i=1}^{n}\left|\alpha_{i}\right| q^{-p_{i}} \eta_{i}^{q}\right) \\
& =\|\varphi\| \Omega \leq \rho .
\end{aligned}
$$

This shows that $\mathcal{A}_{1} x+\mathcal{A}_{2} y \in B_{\rho}$. It is easy to see using (3.5) that $\mathcal{A}_{2}$ is a contraction mapping.

Continuity of $f$ implies that the operator $\mathcal{A}_{1}$ is continuous. Also, $\mathcal{A}_{1}$ is uniformly bounded on $B_{\rho}$ as

$$
\left\|\mathcal{A}_{1} x\right\| \leq \frac{T^{q}}{\Gamma(q+1)}\|\varphi\| .
$$

Now we prove the compactness of the operator $\mathcal{A}_{1}$.

We define $\sup _{(t, x) \in[0, T] \times B_{\rho}}|f(t, x)|=\bar{f}<\infty$, and consequently we have

$$
\begin{aligned}
\left|\left(\mathcal{A}_{1} x\right)\left(t_{2}\right)-\left(\mathcal{A}_{1} x\right)\left(t_{1}\right)\right|= & \frac{1}{\Gamma(q)} \mid \int_{0}^{t_{1}}\left[\left(t_{2}-s\right)^{q-1}-\left(t_{1}-s\right)^{q-1}\right] f(s, x(s)) d s \\
& +\int_{t_{1}}^{t_{2}}\left(t_{2}-s\right)^{q-1} f(s, x(s)) d s \mid \\
\leq & \frac{\bar{f}}{\Gamma(q+1)}\left|t_{1}^{q}-t_{2}^{q}\right|
\end{aligned}
$$

which is independent of $x$ and tend to zero as $t_{2}-t_{1} \rightarrow 0$. Thus, $\mathcal{A}_{1}$ is equicontinuous. So $\mathcal{A}_{1}$ is relatively compact on $B_{\rho}$. Hence, by Arzelá-Ascoli's theorem, $\mathcal{A}_{1}$ is compact on $B_{\rho}$. Thus all the assumptions of Lemma 3.2 are satisfied. So the conclusion of Lemma 3.2 implies that the boundary value problem (1.1)-(1.2) has at least one solution on $[0, T]$.

\subsection{Existence result via Leray-Schauder's nonlinear alternative}

Theorem 3.5 (Nonlinear alternative for single valued maps) [25] Let E be a Banach space, $C$ a closed, convex subset of $E, U$ an open subset of $C$ and $0 \in U$. Suppose that $\mathcal{A}: \bar{U} \rightarrow C$ is a continuous, compact (that is, $\mathcal{A}(\bar{U})$ is a relatively compact subset of $C$ ) map. Then:

(i) either $\mathcal{A}$ has a fixed point in $\bar{U}$, or

(ii) there is a $x \in \partial U$ (the boundary of $U$ in $C$ ) and $\lambda \in(0,1)$ with $x=\lambda \mathcal{A}(x)$.

Theorem 3.6 Assume that:

$\left(\mathrm{H}_{5}\right)$ there exist a continuous nondecreasing function $\psi:[0, \infty) \rightarrow(0, \infty)$ and a function $p \in C\left([0, T], \mathbb{R}^{+}\right)$such that 


$$
|f(t, u)| \leq p(t) \psi(\|x\|) \quad \text { for each }(t, x) \in[0, T] \times \mathbb{R}
$$

$\left(\mathrm{H}_{6}\right)$ there exists a constant $M>0$ such that

$$
\frac{M}{\psi(M)\|p\| \Omega}>1,
$$

where $\Omega$ is defined by (3.2).

Then the boundary value problem (1.1)-(1.2) has at least one solution on [0, T].

Proof Let the operator $\mathcal{A}$ be defined by (3.1). Firstly, we shall show that $\mathcal{A}$ maps bounded sets (balls) into bounded sets in $C([0, T], \mathbb{R})$. For a number $r>0$, let $B_{r}=\{x \in C([0, T], \mathbb{R})$ : $\|x\| \leq r\}$ be a bounded ball in $C([0, T], \mathbb{R})$. Then for $t \in[0, T]$ we have

$$
\begin{aligned}
|(\mathcal{A} x)(t)| \leq & \sup _{t \in[0, T]}\left\{R_{R} I^{q}|f(s, x(s))|(t)+\frac{t^{q-1}}{|\lambda|} R L I^{q}|f(s, x(s))|(T)\right. \\
& \left.+\frac{t^{q-1}}{|\lambda|} \sum_{i=1}^{n}\left|\alpha_{i}\right|_{H} I^{p_{i}}{ }_{R L} I^{q}|f(s, x(s))|\left(\eta_{i}\right)\right\} \\
\leq & \psi(\|x\|)_{R L} I^{q} p(s)(T)+\psi(\|x\|) \frac{T^{q-1}}{|\lambda|} R L I^{q} p(s)(T) \\
& +\psi(\|x\|) \frac{T^{q-1}}{|\lambda|} \sum_{i=1}^{n}\left|\alpha_{i}\right|_{H} I^{p_{i}} R I^{q} p(s)\left(\eta_{i}\right) \\
\leq & \psi(\|x\|)\|p\|\left(\frac{T^{q}}{\Gamma(q+1)}+\frac{T^{2 q-1}}{|\lambda| \Gamma(q+1)}+\frac{T^{q-1}}{|\lambda| \Gamma(q+1)} \sum_{i=1}^{n}\left|\alpha_{i}\right| q^{-p_{i}} \eta_{i}^{q}\right)
\end{aligned}
$$

and consequently,

$$
\|\mathcal{A} x\| \leq \psi(r)\|p\| \Omega
$$

Next we will show that $\mathcal{A}$ maps bounded sets into equicontinuous sets of $C([0, T], \mathbb{R})$. Let $\tau_{1}, \tau_{2} \in[0, T]$ with $\tau_{1}<\tau_{2}$ and $x \in B_{r}$. Then we have

$$
\begin{aligned}
& \left|(\mathcal{A} x)\left(\tau_{2}\right)-(\mathcal{A} x)\left(\tau_{1}\right)\right| \\
& \leq \frac{1}{\Gamma(q)}\left|\int_{0}^{\tau_{1}}\left[\left(\tau_{2}-s\right)^{q-1}-\left(\tau_{1}-s\right)^{q-1}\right] f(s, x(s)) d s+\int_{\tau_{1}}^{\tau_{2}}\left(\tau_{2}-s\right)^{q-1} f(s, x(s)) d s\right| \\
& \quad+\frac{\left(\tau_{2}^{q-1}-\tau_{1}^{q-1}\right)}{|\lambda|} R I^{q}|f(s, x(s))|(T)+\frac{\left(\tau_{2}^{q-1}-\tau_{1}^{q-1}\right)}{|\lambda|} \sum_{i=1}^{n}\left|\alpha_{i}\right|_{H} I^{p_{i}}{ }_{R L} I^{q}|f(s, x(s))|\left(\eta_{i}\right) \\
& \leq \frac{\psi(r)\left|\int_{0}^{\tau_{1}}\left[\left(\tau_{2}-s\right)^{q-1}-\left(\tau_{1}-s\right)^{q-1}\right] p(s) d s+\int_{\tau_{1}}^{\tau_{2}}\left(\tau_{2}-s\right)^{q-1} p(s) d s\right|}{\Gamma(q) \mid} \\
& \quad+\frac{\left(\tau_{2}^{q-1}-\tau_{1}^{q-1}\right) \psi(r)}{|\lambda|}{ }_{R L} I^{q} p(s)(T)+\frac{\left(\tau_{2}^{q-1}-\tau_{1}^{q-1}\right) \psi(r)}{|\lambda|} \sum_{i=1}^{n}\left|\alpha_{i}\right|_{H} I^{p_{i}}{ }_{R L} I^{q} p(s)\left(\eta_{i}\right) .
\end{aligned}
$$


As $\tau_{2}-\tau_{1} \rightarrow 0$, the right-hand side of the above inequality tends to zero independently of $x \in B_{r}$. Therefore by Arzelá-Ascoli's theorem the operator $\mathcal{A}: C([0, T], \mathbb{R}) \rightarrow C([0, T], \mathbb{R})$ is completely continuous.

Let $x$ be a solution. Then, for $t \in[0, T]$, and following similar computations to the first step, we have

$$
|x(t)| \leq \psi(\|x\|)\|p\| \Omega
$$

which leads to

$$
\frac{\|x\|}{\psi(\|x\|)\|p\| \Omega} \leq 1 .
$$

In view of $\left(\mathrm{H}_{6}\right)$, there exists $M$ such that $\|x\| \neq M$. Let us set

$$
U=\{x \in C([0, T], \mathbb{R}):\|x\|<M\} .
$$

We see that the operator $\mathcal{A}: \bar{U} \rightarrow C([0, T], \mathbb{R})$ is continuous and completely continuous. From the choice of $U$, there is no $x \in \partial U$ such that $x=v \mathcal{A} x$ for some $v \in(0,1)$. Consequently, by the nonlinear alternative of Leray-Schauder type, we deduce that $\mathcal{A}$ has a fixed point $x \in \bar{U}$ which is a solution of the boundary value problem (1.1)-(1.2). This completes the proof.

\subsection{Existence result via Leray-Schauder's degree theory}

Theorem 3.7 Let $f:[0, T] \times \mathbb{R} \rightarrow \mathbb{R}$ be a continuous function. Suppose that

$\left(\mathrm{H}_{5}\right)$ there exist constants $0 \leq \kappa<\Omega^{-1}$ and $M>0$ such that

$$
|f(t, x)| \leq \kappa|x|+M \quad \text { for all }(t, x) \in[0, T] \times \mathbb{R}
$$

where $\Omega$ is defined by (3.2).

Then the boundary value problem (1.1)-(1.2) has at least one solution on [0, T].

Proof We define an operator $\mathcal{A}: \mathcal{C} \rightarrow \mathcal{C}$ as in (3.1). In view of the fixed point problem

$$
x=\mathcal{A} x .
$$

We shall prove the existence of at least one solution $x \in C[0, T]$ satisfying (3.7). Set a ball $B_{R} \subset C[0, T]$, as

$$
B_{R}=\left\{x \in \mathcal{C}: \max _{t \in C[0, T]}|x(t)|<R\right\}
$$

where a constant radius $R>0$. Hence, we shall show that $\mathcal{A}: \bar{B}_{R} \rightarrow C[0, T]$ satisfies the condition

$$
x \neq \theta \mathcal{A} x, \quad \forall x \in \partial B_{R}, \forall \theta \in[0,1] .
$$


We set

$$
H(\theta, x)=\theta \mathcal{A} x, \quad x \in \mathcal{C}, \theta \in[0,1] .
$$

As shown in Theorem 3.6 we see that the operator $\mathcal{A}$ is continuous, uniformly bounded, and equicontinuous. Then, by Arzelá-Ascoli's theorem, a continuous map $h_{\theta}$ defined by $h_{\theta}(x)=x-H(\theta, x)=x-\theta \mathcal{A} x$ is completely continuous. If (3.8) holds, then the following Leray-Schauder degrees are well defined, and by the homotopy invariance of topological degree it follows that

$$
\begin{aligned}
\operatorname{deg}\left(h_{\theta}, B_{R}, 0\right) & =\operatorname{deg}\left(I-\theta \mathcal{A}, B_{R}, 0\right)=\operatorname{deg}\left(h_{1}, B_{R}, 0\right) \\
& =\operatorname{deg}\left(h_{0}, B_{R}, 0\right)=\operatorname{deg}\left(I, B_{R}, 0\right)=1 \neq 0, \quad 0 \in B_{R},
\end{aligned}
$$

where $I$ denotes the unit operator. By the nonzero property of the Leray-Schauder degree, $h_{1}(x)=x-\mathcal{A} x=0$ for at least one $x \in B_{R}$. Let us assume that $x=\theta \mathcal{A} x$ for some $\theta \in[0,1]$ and for all $t \in[0, T]$ so that

$$
\begin{aligned}
|x(t)|= & |\theta(\mathcal{A} x)(t)| \\
\leq & { }_{L} I^{q}|f(s, x(s))|(t)+\frac{t^{q-1}}{|\lambda|} R L I^{q}|f(s, x(s))|(T) \\
& +\frac{t^{q-1}}{|\lambda|} \sum_{i=1}^{n}\left|\alpha_{i}\right|_{H} I^{p_{i}}{ }_{R L} I^{q}|f(s, x(s))|\left(\eta_{i}\right) \\
\leq & (\kappa|x|+M)_{R L} I^{q}(1)(T)+(\kappa|x|+M) \frac{T^{q-1}}{|\lambda|} R L^{q}(1)(T) \\
& +(\kappa|x|+M) \frac{T^{q-1}}{|\lambda|} \sum_{i=1}^{n}\left|\alpha_{i}\right|_{H} I^{p_{i}}{ }_{R L} I^{q} p(s)\left(\eta_{i}\right) \\
\leq & (\kappa|x|+M)\left(\frac{T^{q}}{\Gamma(q+1)}+\frac{T^{2 q-1}}{|\lambda| \Gamma(q+1)}+\frac{T^{q-1}}{|\lambda| \Gamma(q+1)} \sum_{i=1}^{n}\left|\alpha_{i}\right| q^{-p_{i}} \eta_{i}^{q}\right) \\
= & (\kappa|x|+M) \Omega,
\end{aligned}
$$

which, on taking the norm $\sup _{t \in[0, T]}|x(t)|=\|x\|$ and solving for $\|x\|$, yields

$$
\|x\| \leq \frac{M \Omega}{1-\kappa \Omega}
$$

If $R=\frac{M \Omega}{1-\kappa \Omega}+1$, inequality (3.8) holds. This completes the proof.

\section{Examples}

In this section, we present some examples to illustrate our results.

Example 4.1 Consider the following nonlocal Hadamard fractional integral conditions for a nonlinear Riemann-Liouville fractional differential equation:

$$
\left\{\begin{array}{l}
{ }_{R L} D^{\frac{3}{2}} x(t)=\frac{\sin ^{2}(\pi t)}{\left(e^{t}+3\right)^{2}} \cdot \frac{|x(t)|}{|x(t)|+1}+\frac{\sqrt{3}}{2}, \quad t \in[0,3], \\
x(0)=0, \quad x(3)+\sqrt{5}_{H} I^{1 / 2} x\left(\frac{9}{4}\right)=\frac{4}{5} H^{\sqrt{2}} x\left(\frac{3}{4}\right)+\frac{\sqrt{3}}{2}{ }_{H} I^{\pi} x\left(\frac{3}{2}\right) .
\end{array}\right.
$$


Here $q=3 / 2, n=3, T=3, \alpha_{1}=4 / 5, \alpha_{2}=\sqrt{3} / 2, \alpha_{3}=-\sqrt{5}, p_{1}=\sqrt{2}, p_{2}=\pi, p_{3}=1 / 2$, $\eta_{1}=3 / 4, \eta_{2}=3 / 2, \eta_{3}=9 / 4$, and $f(t, x)=\left(\sin ^{2}(\pi t) /\left(e^{t}+3\right)^{2}\right)(|x| /(1+|x|))+(\sqrt{3} / 2)$. Since $|f(t, x)-f(t, y)| \leq(1 / 16)|x-y|,\left(\mathrm{H}_{1}\right)$ is satisfied with $L=1 / 16$. By using a Maple program, we can find that

$$
\Omega:=\frac{T^{q}}{\Gamma(q+1)}+\frac{T^{2 q-1}}{|\lambda| \Gamma(q+1)}+\frac{T^{q-1}}{|\lambda| \Gamma(q+1)} \sum_{i=1}^{n}\left|\alpha_{i}\right| q^{-p_{i}} \eta_{i}^{q} \approx 7.239901027
$$

Thus $L \Omega \approx 0.4524938142<1$. Hence, by Theorem 3.1, the boundary value problem (4.1) has a unique solution on $[0,3]$.

Example 4.2 Consider the following nonlocal Hadamard fractional integral conditions for a nonlinear Riemann-Liouville fractional differential equation:

$$
\left\{\begin{array}{l}
R L D^{\frac{4}{3}} x(t)=\frac{e^{t}}{e^{t}+8} \cdot \frac{|x(t)|}{|x(t)|+2}+1, \quad t \in\left[0, \frac{3}{2}\right], \\
x(0)=0, \\
x\left(\frac{3}{2}\right)+\frac{2}{3} I^{\sqrt{2} / 2} x\left(\frac{3}{5}\right)+\pi_{H} I^{\sqrt{3}} x\left(\frac{6}{5}\right)=\frac{1}{5}{ }_{H} I^{1 / 4} x\left(\frac{3}{10}\right)+\frac{1}{\sqrt{3}} H^{6 / 5} x\left(\frac{9}{10}\right) .
\end{array}\right.
$$

Here $q=4 / 3, n=4, T=3 / 2, \alpha_{1}=1 / 5, \alpha_{2}=-2 / 3, \alpha_{3}=1 / \sqrt{3}, \alpha_{4}=-\pi / 2, p_{1}=1 / 4$, $p_{2}=\sqrt{2} / 2, p_{3}=6 / 5, p_{4}=\sqrt{3}, \eta_{1}=3 / 10, \eta_{2}=3 / 5, \eta_{3}=9 / 10$, and $\eta_{4}=6 / 5$. Since $\mid f(t, x)-$ $f(t, y)\left|\leq\left(2 e^{t} /\left(e^{t}+8\right)\right)\right| x-y \mid$, then $\left(\mathrm{H}_{2}\right)$ is satisfied with $\delta(t)=2 e^{t} /\left(e^{t}+8\right)$ and $\sigma=1 / 2$. By using a Maple program, we can show that

$$
\begin{aligned}
& \|\delta\|\left\{\frac{T^{q-\sigma}}{\Gamma(q)}\left(\frac{1-\sigma}{q-\sigma}\right)^{1-\sigma}+\frac{T^{2 q-\sigma-1}}{|\lambda| \Gamma(q)}\left(\frac{1-\sigma}{q-\sigma}\right)^{1-\sigma}\right. \\
& \left.+\frac{T^{q-1}}{|\lambda| \Gamma(q)}\left(\frac{1-\sigma}{q-\sigma}\right)^{1-\sigma} \sum_{i=1}^{n}\left|\alpha_{i}\right|(q-\sigma)^{p_{i}} \eta_{i}^{q-\sigma}\right\} \approx 0.9380422264<1 .
\end{aligned}
$$

Hence, by Theorem 3.2, the boundary value problem (4.2) has a unique solution on $[0,3 / 2]$.

Example 4.3 Consider the following nonlocal Hadamard fractional integral conditions for a nonlinear Riemann-Liouville fractional differential equation:

$$
\left\{\begin{array}{l}
{ }_{R L} D^{\frac{7}{6}} x(t)=\frac{t^{2}}{(t+2)^{2}} \cdot \frac{|x(t)|}{|x(t)|+1}+3 t+\frac{4}{5}, \quad t \in[0,2], \\
x(0)=0, \quad x(2)=2_{H} I^{\sqrt{\pi}} x\left(\frac{2}{5}\right)+\frac{2}{3} H^{5 / 4} x\left(\frac{4}{3}\right)+\sqrt{3}_{H} I^{3 / 7} x\left(\frac{3}{2}\right) .
\end{array}\right.
$$

Here $q=7 / 6, n=3, T=2, \alpha_{1}=2, \alpha_{2}=2 / 3, \alpha_{3}=\sqrt{3}, p_{1}=\sqrt{\pi}, p_{2}=5 / 4, p_{3}=3 / 7, \eta_{1}=2 / 5$, $\eta_{2}=4 / 3, \eta_{3}=3 / 2$, and $f(t, x)=\left(t^{2}|x| /\left((t+2)^{2}\right)(|x|+1)\right)+3 t+(4 / 5)$. We choose $h(t)=t^{2} / 4$ and

$$
\begin{aligned}
H^{*} & :={ }_{R L} I^{q} h(T)+\frac{T^{q-1}}{|\lambda|} R L I^{q} h(T)+\frac{T^{q-1}}{|\lambda|} \sum_{i=1}^{n}\left|\alpha_{i}\right|_{H} I^{p_{i}}{ }_{R L} I^{q} h\left(\eta_{i}\right) \\
& \approx 0.6432886158 .
\end{aligned}
$$


Clearly,

$$
|f(t, x)-f(t, y)|=\frac{t^{2}}{(t+2)^{2}}\left|\frac{|x|-|y|}{1+|x|+|y|+|x||y|}\right| \leq \frac{t^{2}}{4}\left(\frac{|x-y|}{0.6432886158+|x-y|}\right) .
$$

Hence, by Theorem 3.3, the boundary value problem (4.3) has a unique solution on $[0,2]$.

Example 4.4 Consider the following nonlocal Hadamard fractional integral conditions for a nonlinear Riemann-Liouville fractional differential equation:

$$
\left\{\begin{array}{l}
R L^{\frac{5}{4}} x(t)=\frac{e^{-t^{2}} \sin ^{2}(2 t)}{(t+3)^{2}} \cdot \frac{|x(t)|}{|x(t)|+1}+\frac{t-1}{t+1}, \quad t \in[0,2 \pi], \\
x(0)=0, \\
x(2 \pi)+\sqrt{3}{ }_{H} I^{1 / 2} x\left(\frac{\pi}{3}\right)+\frac{3}{4} H I^{3 / 4} x\left(\frac{2 \pi}{3}\right)={ }_{H} I^{4 / 5} x(\pi)+\frac{1}{9} H_{H} I^{4 / 3} x\left(\frac{4 \pi}{3}\right)+2{ }_{H} I^{2 / 3} x\left(\frac{5 \pi}{3}\right) .
\end{array}\right.
$$

Here $q=5 / 4, n=5, T=2 \pi, \alpha_{1}=-\sqrt{3}, \alpha_{2}=-3 / 4, \alpha_{3}=1, \alpha_{4}=1 / 9, \alpha_{5}=2, p_{1}=1 / 2$, $p_{2}=3 / 4, p_{3}=4 / 5, p_{4}=4 / 3, p_{5}=2 / 3, \eta_{1}=\pi / 3, \eta_{2}=2 \pi / 3, \eta_{3}=\pi, \eta_{4}=4 \pi / 3, \eta_{5}=5 \pi / 3$, and $f(t, x)=\left(e^{-t^{2}} \sin ^{2}(2 t)|x|\right) /\left(\left((t+3)^{2}\right)(|x|+1)\right)+(t-1) /(t+1)$. Since $|f(t, x)-f(t, y)| \leq$ $(1 / 9)|x-y|,\left(\mathrm{H}_{1}\right)$ is satisfied with $L=1 / 36$. By a Maple program, we show that

$$
L\left(\frac{T^{2 q-1}}{|\lambda| \Gamma(q+1)}+\frac{T^{q-1}}{|\lambda| \Gamma(q+1)} \sum_{i=1}^{n}\left|\alpha_{i}\right| q^{-p_{i}} \eta_{i}^{q}\right) \approx 0.9518560542<1 .
$$

Clearly,

$$
|f(t, x)|=\left|\frac{e^{-t^{2}} \sin ^{2}(2 t)}{(t+3)^{2}} \cdot \frac{|x(t)|}{|x(t)|+1}+\frac{t-1}{t+1}\right| \leq \frac{e^{-t^{2}}}{9}+\frac{t-1}{t+1} .
$$

Hence, by Theorem 3.4, the boundary value problem (4.4) has at least one solution on $[0,2 \pi]$.

Example 4.5 Consider the following nonlocal Hadamard fractional integral conditions for a nonlinear Riemann-Liouville fractional differential equation:

$$
\left\{\begin{array}{l}
{ }_{R} D^{\frac{6}{5}} x(t)=\frac{1}{64}\left(1+t^{2}\right)\left(\frac{x^{2}}{|x|+1}+\frac{\sqrt{|x|}}{2(1+\sqrt{|x|)}}+\frac{1}{2}\right), \quad t \in[0, e], \\
x(0)=0, \quad x(e)=\frac{1}{2} I^{\sqrt{2}} x\left(\frac{1}{2}\right)-5_{H} I^{\sqrt{3}} x\left(\frac{2}{3}\right)+\sqrt{3}_{H} I^{\sqrt{5}} x(1) .
\end{array}\right.
$$

Here $q=6 / 5, n=3, T=e, \alpha_{1}=1 / 2, \alpha_{2}=-5, \alpha_{3}=\sqrt{3}, p_{1}=\sqrt{2}, p_{2}=\sqrt{3}, p_{3}=\sqrt{5}, \eta_{1}=1 / 2$, $\eta_{2}=2 / 3, \eta_{3}=1$, and $f(t, x)=(1 / 64)\left(1+t^{2}\right)\left(\left(x^{2} /(|x|+1)\right)+(\sqrt{x}) /(2(1+\sqrt{x}))+(1 / 2)\right)$. It is easy to verify that

$$
\Omega:=\frac{T^{q}}{\Gamma(q+1)}+\frac{T^{2 q-1}}{|\lambda| \Gamma(q+1)}+\frac{T^{q-1}}{|\lambda| \Gamma(q+1)} \sum_{i=1}^{n}\left|\alpha_{i}\right| q^{-p_{i}} \eta_{i}^{q} \approx 3.905177250 .
$$

Clearly,

$$
|f(t, x)|=\left|\frac{1}{64}\left(1+t^{2}\right)\left(\frac{x^{2}}{|x|+1}+\frac{\sqrt{|x|}}{2(1+\sqrt{|x|})}+\frac{1}{2}\right)\right| \leq \frac{1}{64}\left(1+t^{2}\right)(|x|+1) .
$$


Choosing $p(t)=(1 / 64)\left(1+t^{2}\right)$ and $\psi(|x|)=|x|+1$, we can show that

$$
\frac{M}{\psi(M)\|p\| \Omega}>1
$$

which implies that $M>1.048704821$. Hence, by Theorem 3.6, the boundary value problem (4.5) has at least one solution on $[0, e]$.

Example 4.6 Consider the following nonlocal Hadamard fractional integral conditions for a nonlinear Riemann-Liouville fractional differential equation:

$$
\left\{\begin{array}{l}
{ }_{R} D^{\frac{7}{4}} x(t)=\frac{1}{2 \pi} \sin \left(\frac{\pi}{2} x\right) \cdot \frac{|x|}{|x|+1}+1, \quad t \in[0,1], \\
x(0)=0, \quad x(1)=3_{H} I^{1 / 2} x\left(\frac{1}{2}\right)-2{ }_{H} I^{3 / 2} x\left(\frac{3}{4}\right)
\end{array}\right.
$$

Here $q=7 / 4, n=2, T=1, \alpha_{1}=3, \alpha_{2}=-2, p_{1}=1 / 2, p_{2}=3 / 2, \eta_{1}=1 / 2, \eta_{2}=3 / 4$, and $f(t, x)=(1 / 2 \pi)(\sin (\pi x / 2))(|x| /(|x|+1))+1$. We can show that

$$
\Omega:=\frac{T^{q}}{\Gamma(q+1)}+\frac{T^{2 q-1}}{|\lambda| \Gamma(q+1)}+\frac{T^{q-1}}{|\lambda| \Gamma(q+1)} \sum_{i=1}^{n}\left|\alpha_{i}\right| q^{-p_{i}} \eta_{i}^{q} \approx 1.582207843
$$

Since

$$
|f(t, x)|=\left|\frac{1}{2 \pi} \sin \left(\frac{\pi}{2} x\right) \cdot \frac{|x|}{|x|+1}+1\right| \leq \frac{1}{4}|x|+1,
$$

$\left(\mathrm{H}_{5}\right)$ is satisfied with $\kappa=1 / 4$ and $M=1$ such that

$$
\kappa=\frac{1}{4}<\frac{1}{\Omega} \approx 0.6320282158
$$

Hence, by Theorem 3.7, the boundary value problem (4.6) has at least one solution on $[0,1]$.

\section{Competing interests}

The authors declare that they have no competing interests.

\section{Authors' contributions}

All authors contributed equally in this article. They read and approved the final manuscript.

\section{Author details}

${ }^{1}$ Nonlinear Dynamic Analysis Research Center, Department of Mathematics, Faculty of Applied Science, King Mongkut's University of Technology North Bangkok, Bangkok, 10800, Thailand. ²Department of Mathematics, University of loannina, loannina, 451 10, Greece.

\section{Authors' information}

Sotiris K Ntouyas is a member of Nonlinear Analysis and Applied Mathematics (NAAM)-Research Group at King Abdulaziz University, Jeddah, Saudi Arabia.

\section{Acknowledgements}

This research was funded by King Mongkut's University of Technology North Bangkok, Thailand. Contract no. KMUTNB-GEN-58-09. 


\section{References}

1. Podlubny, I: Fractional Differential Equations. Academic Press, San Diego (1999)

2. Kilbas, AA, Srivastava, HM, Trujillo, JJ: Theory and Applications of Fractional Differential Equations. North-Holland Mathematics Studies, vol. 204. Elsevier, Amsterdam (2006)

3. Baleanu, D, Diethelm, K, Scalas, E, Trujillo, JJ: Fractional Calculus Models and Numerical Methods. Series on Complexity, Nonlinearity and Chaos. World Scientific, Boston (2012)

4. Agarwal, RP, Zhou, Y, He, Y: Existence of fractional neutral functional differential equations. Comput. Math. Appl. 59, 1095-1100 (2010)

5. Baleanu, D, Mustafa, OG, Agarwal, RP: On $L^{p}$-solutions for a class of sequential fractional differential equations. Appl. Math. Comput. 218, 2074-2081 (2011)

6. Ahmad, B, Nieto, JJ: Riemann-Liouville fractional integro-differential equations with fractional nonlocal integral boundary conditions. Bound. Value Probl. 2011, Article ID 36 (2011)

7. Ahmad, B, Ntouyas, SK, Alsaedi, A: New existence results for nonlinear fractional differential equations with three-point integral boundary conditions. Adv. Differ. Equ. 2011, Article ID 107384 (2011)

8. O'Regan, D, Stanek, S: Fractional boundary value problems with singularities in space variables. Nonlinear Dyn. 71, 641-652 (2013)

9. Ahmad, B, Ntouyas, SK, Alsaedi, A: A study of nonlinear fractional differential equations of arbitrary order with Riemann-Liouville type multistrip boundary conditions. Math. Probl. Eng. 2013, Article ID 320415 (2013)

10. Ahmad, B, Nieto, JJ: Boundary value problems for a class of sequential integrodifferential equations of fractional order. J. Funct. Spaces Appl. 2013, Article ID 149659 (2013)

11. Zhang, L, Ahmad, B, Wang, G, Agarwal, RP: Nonlinear fractional integro-differential equations on unbounded domains in a Banach space. J. Comput. Appl. Math. 249, 51-56 (2013)

12. Liu, X, Jia, M, Ge, W: Multiple solutions of a $p$-Laplacian model involving a fractional derivative. Adv. Differ. Equ. 2013 Article ID 126 (2013)

13. Sudsutad, W, Tariboon, J: Boundary value problems for fractional differential equations with three-point fractional integral boundary conditions. Adv. Differ. Equ. 2012, Article ID 93 (2012)

14. Sudsutad, W, Tariboon, J: Existence results of fractional integro-differential equations with $m$-point multi-term fractional order integral boundary conditions. Bound. Value Probl. 2012, Article ID 94 (2012)

15. Hadamard, J: Essai sur l'étude des fonctions données par leur développement de Taylor. J. Math. Pures Appl. 8 101-186 (1892)

16. Butzer, PL, Kilbas, AA, Trujillo, JJ: Compositions of Hadamard-type fractional integration operators and the semigroup property. J. Math. Anal. Appl. 269, 387-400 (2002)

17. Butzer, PL, Kilbas, AA, Trujillo, JJ: Fractional calculus in the Mellin setting and Hadamard-type fractional integrals. J. Math. Anal. Appl. 269, 1-27 (2002)

18. Butzer, PL, Kilbas, AA, Trujillo, JJ: Mellin transform analysis and integration by parts for Hadamard-type fractional integrals. J. Math. Anal. Appl. 270, 1-15 (2002)

19. Kilbas, AA: Hadamard-type fractional calculus. J. Korean Math. Soc. 38, 1191-1204 (2001)

20. Kilbas, AA, Trujillo, JJ: Hadamard-type integrals as G-transforms. Integral Transforms Spec. Funct. 14, $413-427$ (2003)

21. Jarad, F, Abdeljawad, T, Baleanu, D: Caputo-type modification of the Hadamard fractional derivatives. Adv. Differ. Equ. 2012, Article ID 142 (2012)

22. Gambo, YY, Jarad, F, Baleanu, D, Abdeljawad, T: On Caputo modification of the Hadamard fractional derivatives. Adv. Differ. Equ. 2014, Article ID 10 (2014)

23. Boyd, DW, Wong, JSW: On nonlinear contractions. Proc. Am. Math. Soc. 20, 458-464 (1969)

24. Krasnoselskii, MA: Two remarks on the method of successive approximations. Usp. Mat. Nauk 10, $123-127$ (1955)

25. Granas, A, Dugundji, J: Fixed Point Theory. Springer, New York (2003)

doi:10.1186/s13661-014-0253-9

Cite this article as: Tariboon et al.: Nonlocal Hadamard fractional integral conditions for nonlinear Riemann-Liouville fractional differential equations. Boundary Value Problems 2014 2014:253.

\section{Submit your manuscript to a SpringerOpen ${ }^{\circ}$ journal and benefit from:}

- Convenient online submission

- Rigorous peer review

- Immediate publication on acceptance

- Open access: articles freely available online

- High visibility within the field

- Retaining the copyright to your article 